\title{
Research on the Design Method of the High-speed Motor Based on the Ultrasonic Levitation Support
}

\author{
Wang Hongchen and Yang Zhigang \\ School of Mechanical Science and Engineering of Jilin University, Changchun, \\ Jilin, 130025, China \\ Engineering Training Center of Changchun Institute of Technology, Changchun, \\ Jilin, 130012, China \\ School of Mechanical Science and Engineering of Jilin University, Changchun, \\ Jilin, 130025, China \\ whcwanghongchen@126.com,yzg@jlu.edu.cn
}

\begin{abstract}
This essay presents the design method of applying ultrasonic levitation bearing to high-speed motor and using single supporting rotor of ultrasonic levitation high-speed motor. It adopts piezoelectric transducer, the permanent magnet synchronous motor spindle; the radiation surface of transducer and the levitation support cap of the spindle adopt the conical structure with the same taper. It ensures that the spindle is highly automatic centering when it rotates at the high speed. The test results show that the spindle runs smoothly without having contact friction noise and problems of torque fluctuation and radial vibration caused by the electromagnetic coupling, when the output power of the ultrasonic power supply is at 500w, the speed of the spindle is over $20000 \mathrm{rpm}$, and the levitation clearance is more than 100nm, which is more than the sum of roughness Ra for two contact surfaces.
\end{abstract}

Keywords: Ultrasonic, Suspension bearing, High speed motor, Piezoelectric transducer, Single support, Suspension clearance, Spindle, Cone structure

\section{Introduction}

High-speed motor is widely used in industrial fields, such as high-speed machine tool, the turbo molecular pump, high-speed centrifuge, precision gyroscope, and flywheel energy storage. It shows its increasingly important and broad application prospects. The rotor of the traditional high speed motor is supported by two mechanical bearings, which has great friction and wear, low life, poor reliability, and produces serious mechanical vibration and noise. This has been unable to meet the supporting requirements of high speed motor. Current methods to solve this problem are to use the non-contact air, liquid or magnetic bearing, which can basically achieve the low friction or friction free operation, and increase the motor speed to the level of over 5000r/min. However, it also brings some problems, such as the complex structure of the system, high energy consumption, low efficiency, large volume, and the complex control system. These problems seriously restrict the further development and application of high-speed motor [1-4]. In recent years, some people proposed the bearingless motor technology. The current research work is mainly focused on experimental research, technology development, and proving the feasibility of the application of motors with various structures in the bearingless motor technology. It is still in the stage of exploration and research for international studies of the bearingless motor.

In the process of the research on the surface friction properties of the ultrasonic vibration object, it shows that the surface of the ultrasonic vibration object can reduce the 
friction factor of the contact surface, and if the amplitude is large enough, it can suspend the object for a certain distance.

Based on this, it is proposed in this essay to solve the problem of the levitation support of motor rotor by using the ultrasonic levitation supporting technique. This essay studies the basic theory and the key technological problems in the process of this support, tests the levitation support of motor rotor via experimental devices and the stability of high speed rotation, thus forms a new technique or method for the structure of high speed motor, and then lays the foundation of developing a new type of high speed motor with more practical value.

\section{Ultrasonic Levitation and Antifriction}

Ultrasonic levitation technology was first found by Kundt in 1886. Since the 1980s, the scientists all over the world have carried out in-depth studies on the ultrasonic levitation technique and its application. The intensive studies on the relationship between ultrasonic amplitude and friction factor have been conducted by Piezoelectric drive and Control Technology Laboratory of Jilin University [5-13]. The amplitude measurement device is shown in Figure 1 and the results show that:

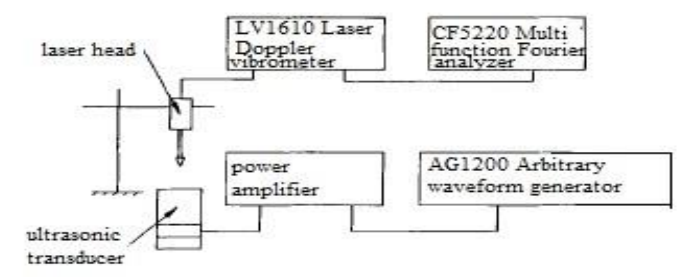

Figure 1. The Amplitude Measurement Device

(1) When the ultrasonic vibration amplitude is less than the sum of the surface roughness $\mathrm{Ra}$ of two contact surfaces, it is performed as the friction reduction effect. It means to decrease the friction factor of two surfaces can reduce the friction factor to the $10 \%$ of the static friction state, so that greatly reduce the frictional wear between the contact surfaces. The curves for the relationship between amplitude and friction factor is shown in Figure 2.

(2) When the ultrasonic vibration amplitude is more than the sum of the surface roughness $\mathrm{Ra}$ of two contact surfaces, it is performed as the suspension effect. It means the larger the amplitude the greater the clearance. When the ultrasonic vibration amplitude is large enough, it can form the absolute suspension on the object. The levitation effect is shown in Figure 3. This can be used to construct various ultrasonic levitation supporting structures for non-contact support in special occasions.

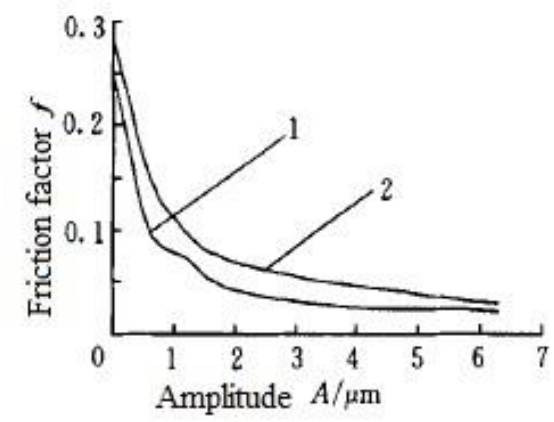

Figure 2. Curves for the Relationship between Amplitude and Friction Factor 


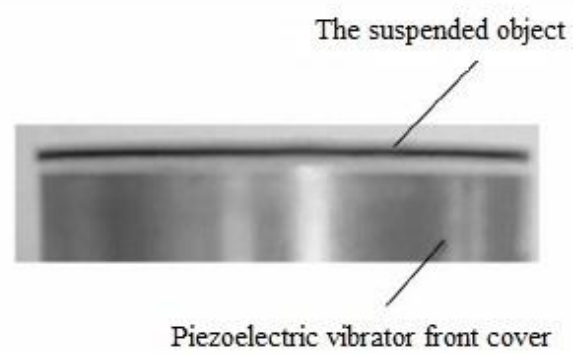

Figure 3. Schematic Diagram of Ultrasonic Levitation Effect (Weight 30g, Levitation Clearance $1.5 \mathrm{~mm}$ )

As long as there is a certain levitation force, it can form the levitation support. Currently, the levitation force density of the superconducting magnetic levitation bearing can reach about $8 \mathrm{~N} / \mathrm{cm}^{2}$. The research results show that the density of the levitation force formed by the near-field acoustic levitation (the levitation clearance is dozens of microns) can reach about $8.5 \mathrm{~N} / \mathrm{cm}^{2}$. It indicates the ultrasonic levitation technology has the research value and wide prospect in the application of the high-speed motor spindle levitation.

3. The Design, Principles, and Functional Parameters about the HighSpeed Electric Spindle of the Ultrasonic Levitation Bearing

\subsection{Electric Spindle}

It adopts the permanent magnet synchronous motor as the research purposes. Electric spindle appearance is shown in Figure 4:

Rated power: $0.3 \mathrm{KW}$

Rated speed: 60000rpm

The quality of the rotor is $200 \mathrm{~g}$.

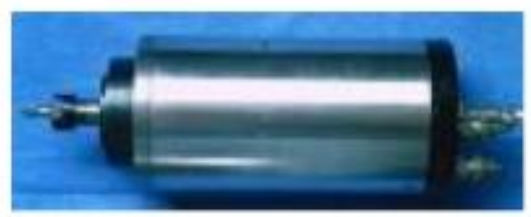

Figure 4. Electric Spindle Appearance

3.2. The Frequency Voltage Curves for the Electric Spindles of Constant Torque and Constant Power

As shown in Figure 5 and Figure 6:

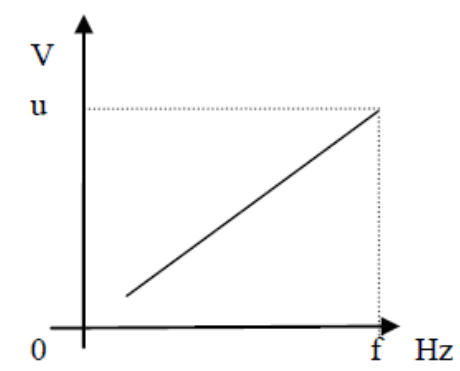

Figure 5. Curve of Constant Torque 


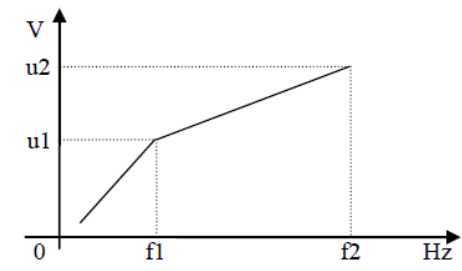

Figure 6. Curve of Constant Power

\subsection{The Fundamental Structure and Operational Principles of Electric Spindle}

An electric spindle is formed by the spindle and the body of the spindle box, the builtin AC servo motor, auxiliary device, and detection device. The rotor of the motor and the spindle are made as a whole part, and the spindle is supported by the front and back spindles. The rotor and the stator are mounted in the casing of the spindle unit through the cooling jackets. The speed change of the spindle is controlled by the driver module of the spindle, and the temperature rise in the spindle unit is controlled by the cooling device. The stator of the electric spindle is stacked by the high-quality silicon steel sheets with high magnetic permeability. The inner cavity of the stack-molding stator has punching inserting groove. The rotor is the rotating part of the intermediate frequency motor. Its function is to convert the electromagnetic energy of the stator into mechanical energy. The rotor is formed by rotor core, rotor cage, and rotation shaft.

\section{The Design and Production of the Test Prototype of the Ultrasonic Levitation Electric Spindle}

\subsection{The Design of Piezoelectric Transducer}

The piezoelectric transducer can effectively realize the conversion between mechanical and electric energy. In the condition of ultrasonic vibration, the surface of the piezoelectric vibrator, especially the output end, has the strong levitation power. This is the premise of the research in this paper. The design framework of the transducer should be able to meet the following two basic requirements:

(1) To achieve the rigid levitation support to the electric main shaft;

(2) To have the stability of the vibration output working in the ultrasonic frequency.

In this paper, the piezoelectric transducer adopted for the study is used for the levitation support to the high-speed shaft. It requires the achievement of energy conversion of high power and high efficiency. When we have designed, an overall consideration has been taken about the power density of the piezoelectric ceramic group, and the material selections to the front and back ends of the piezoelectric transducer and the piezoelectric ceramics. The material for the front end cover of the vibrator is the magnalium with good thermal conductivity and vibration transmission characteristics; the back end cover uses No. 45 steel so that is good for the vibration displacement of the piezoelectric vibrator and the more effective output of the vibrating mechanical energy from the front end. The material for the piezoelectric ceramics should be low in the mechanical and dielectric loss and high for the piezoelectric constant and electromechanical conversion coefficient. Therefore, we have chosen the emission type of the piezoelectric ceramics PZT-8. Through the comparison of the various structures of piezoelectric vibrators, we have adopted the conical piezoelectric transducer to conduct the levitation support to the electric spindle in this experiment. Its structure is shown in Figure 7, and the ultrasonic instrument that works with it is shown in Figure 8. 


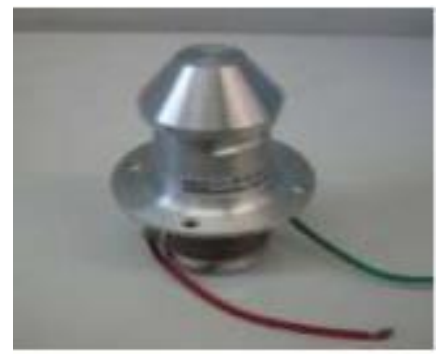

\section{Figure 7. The Conical Piezoelectric Transducer}

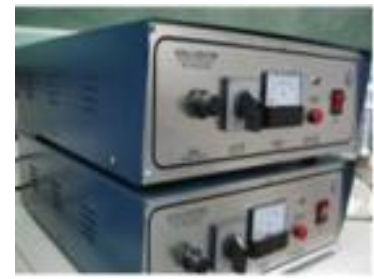

Figure 8. The Ultrasonic Instrument

\subsection{The Test for the Levitation Force of the Piezoelectric Transducer}

We have studied the axial supporting levitation force of single vibrator for the piezoelectric transducer (the end of the transducer is a conical surface). The specific data is shown in Table 1. When the piezoelectric transducer is in the resonant state, the main shaft of the motor $(200 \mathrm{~g})$ is suspended to a certain height, and there is the squeeze film caused by the air between the two matched conical surfaces. The friction resistance at this moment is only caused by the friction of the air and the friction factor is very small, so that the main shaft can achieve a quick and smooth rotation. When the ultrasonic vibration stops, the speed of the main shaft becomes zero rapidly. The friction resistance at this moment is caused by the metal friction of the matched conical surfaces.

Table 1. The Experimental Data for the Test of Single Vibrator Levitation Support with the Conical Surface of Transducer End

\begin{tabular}{|c|c|c|c|c|}
\hline Power & $500(\%)$ & $600(w)$ & $800(w)$ & $1000(w)$ \\
\hline $\begin{array}{l}\text { Levitation } \\
\text { clearance } \\
(\mathrm{mm})\end{array}$ & 0.05 & 0.07 & 0.12 & 0.15 \\
\hline \\
$\begin{array}{l}\text { Note: Power means the output power of the ultrasonic power supply; the } \\
\text { listing figures are the relative heights of the suspendad loads (unit mm) }\end{array}$
\end{tabular}

The experimental study shows: in the state of ultrasonic vibration, the single vibrator with the conical surface of transducer end can achieve the levitation support to the shafting (electric spindle). It has strong bearing capacity and stable operation.

\subsection{The Design and Production of the Prototype of the Ultrasonic Levitation Single Supporting Electric Spindle}

In this paper, we design the overall structure of ultrasonic levitation single support, which keeps the agreement of the tapers of the ultrasonic piezoelectric transducer and the levitation supporting cap of the motor shaft. This structure ensures that the transducer and the electric spindle are strictly coaxial, and also ensures the stability of the levitation support for the electric spindle at high-speed rotation. Its planar structure diagram is shown in Figure 9, and the graphic model is shown in Figure 10. 


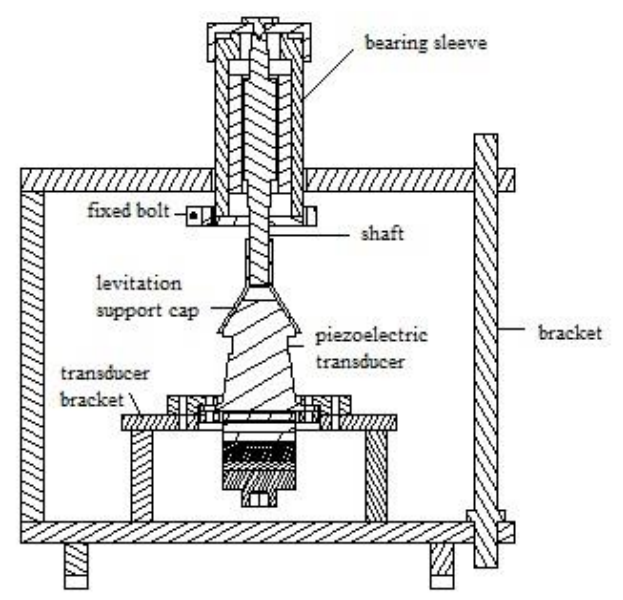

Figure 9. Diagram of the Ultrasonic Axial Supporting Levitation Structure

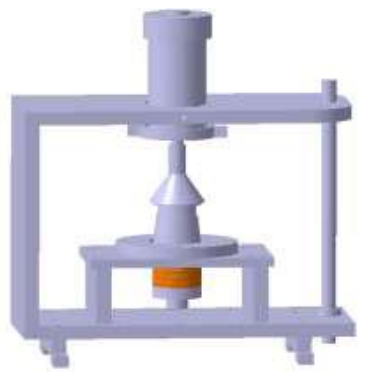

\section{Figure 10. Graphic Model of the Ultrasonic Levitation Axial Support}

Figure 11 shows the test prototype for the electric spindle of the ultrasonic levitation single support with frequency control. There is also a test to the rotation speed, levitation clearance, and stability of the prototype. On the anti-vibration table, when the output power of the ultrasonic power supply is $500 \mathrm{w}$, by using infrared velocimetry and laser micrometer, we have measured that the speed of the spindle is over 20000rpm, the levitation clearance is more than $100 \mathrm{~nm}$, which is more than the sum of roughness Ra for two contact surfaces. The spindle runs smoothly without having contact friction noise.

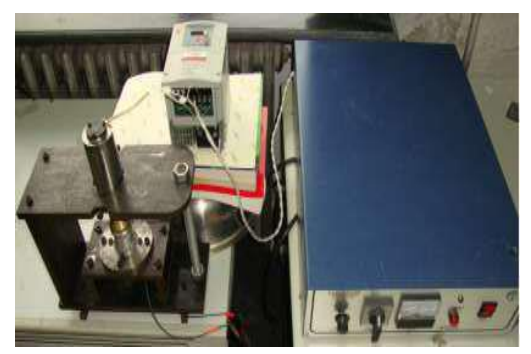

\section{Figure 11. The Test Prototype for the Electric Spindle of the Ultrasonic} Levitation Single Support With Frequency Control

\section{Conclusion}

To sum up, we can draw the following conclusions:

(1) The ultrasonic levitation bearing can replace the traditional bearing, flotation and liquid bearing and apply to the high-speed motor. When the amplitude is larger than $100 \mathrm{~nm}$, it is completely in the state of friction free;

(2) The ultrasonic levitation support is produced by using the mechanical vibration of the 
ultrasonic vibrator. It has no electromagnetic coupling with rotor and stator of motor, so that there is no effect on the motor field. Therefore, it fundamentally eliminates the torque fluctuation and radial vibration problems of magnetic levitation motor caused by the electromagnetic coupling;

(3) The ultrasonic levitation bearing can provide enough preset clearance and have enough support force to achieve the levitation support when the shaft is in the startover and operation. It ensures that the shaft has good centering and stability of rotation.

\section{References}

[1] Y. Zhou, S. Jiang and L. Zhao, "Progress of research on flywheel energy storasystem", Cryogenics and superconductivity, vol.31, no. 1, (2003).

[2] Q. W. Qu, X. H. Li and J. Zhu, "Ultra-thin film lubrication radial bearing simulation tester", Lubrication Engineering, vol.3, no. 18, (1997).

[3] J. Mo, "A study of air squeeze-film damping [J]", Acta Acustica, vol. 16, no. 2, (1991)

[4] L. Chen, Y. Zhang and Y. Xie, "The Development and application of magnetic bearing [J], Journal of Northwest Institute of Light Industry, vol. 9, no. 12, (1996).

[5] Y. Chang, Z. Yang and B. Wu, "Experimental study on bearing capacity and suspension performance of Ultrasonic Bearing [J]", Piezoelectric \& acoustooptic, vol. 27, no. 2, (2004).

[6] Y. Chang, B. Wu and Z. Yang, "The antifriction performance ability and the ultrasonic suspension thrust bearing [J]", Journal of Jilin University, vol. 34, no.2, (2004).

[7] P. Zeng, Y. Cao and G. Cheng, "The mechanism analysis of friction reduction of Ultrasonic Bearing [J]", Lubrication and seal, vol. 182, no. 10, (2006).

[8] Y. Chang, B. Wu and G. Cheng, "Piezoelectric modal analysis and experimental study of Ultrasonic Bearing [J]", Journal of Harbin Institute of Technology, vol. 68, no.5, (2006).

[9] B. Wu, Y. Chang and Z. Yang, "Theoretical analysis and experimental study of ultrasonic vibration anti-friction performance", China Mechanical Engineering, vol. 15, no. 9, (2004)

[10] T. Peng, Z. Yang, F. Tian, "Technology of ultrasonic levitation supporting shaft [J]", Optics and precision engineering, vol. 16, no. 10, (2008).

[11] G. Cheng, P. Zeng, X. Qiu, "Study on ultrasonic vibration antifriction phenomenon [J], Piezoelectrics \& Acoustooptics, vol. 20, no. 5, (1998).

[12] E. Matsuo, Y. Koike, K. Nakamura and S. Ueha, "Holding characteristics of planar objects suspended by near-field acoustic levitation [J],Ultrasonics, vol. 38, no. 5, (2000).

[13] M. Huang, T. Zhou, Q. Wu. "Effect of ultrasonic vibration on the frictional force [J], Chinese Journal of acoustics, vol. 25 , no.2, (2000).

[14] L. Liu, "Design of Motor Rotor Support Structure and Rotor Dynamics Study Based on Ultrasonic Levitation Technology", Jilin University, China (2012).

[15] F. Tian, "Research on Supporting Technique about Ultrasonic Near-field acoustic levitation based on Sandwich Piezoelectric Transducer", Jilin University. China, (2012).

[16] G. Zhou, "The theory of fluid lubrication", Zhejiang University press, (1990).

[17] B. Wen and S. Xia, "Advanced rotor dynamics theory, and should be", Machinery Industry Press, (2000).

\section{Author}

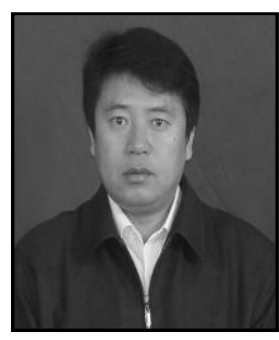

Wang Hong-chen. Male, he was born in May 1972, Jilin University doctoral student, is mainly engaged in the piezo drive and control technology, small machinery and precision machinery research, E-mail: whcwanghongchen@126.com Tel: +8615526639260 . 
International Journal of Control and Automation

Vol. 9, No. 4 (2016) 